\title{
A family of splice variants of CstF-64 expressed in vertebrate nervous systems
}

\author{
Ganesh S Shankarling, Penelope W Coates, Brinda Dass and \\ Clinton C MacDonald*
}

Address: Department of Cell Biology and Biochemistry, Texas Tech University Health Sciences Center, Lubbock, Texas, 79430-6540, USA

Email: Ganesh S Shankarling - ganesh.shankarling@ttuhsc.edu; Penelope W Coates - penelope.coates@ttuhsc.edu; Brinda Dass - brinda.dass@ttuhsc.edu; Clinton C MacDonald* - clint.macdonald@ttuhsc.edu

* Corresponding author

Published: 12 March 2009

BMC Molecular Biology 2009, 10:22 doi:10.1 186/147/-2199-10-22
Received: 31 July 2008

Accepted: 12 March 2009

This article is available from: http://www.biomedcentral.com/I47I-2/99//0/22

(c) 2009 Shankarling et al; licensee BioMed Central Ltd.

This is an Open Access article distributed under the terms of the Creative Commons Attribution License (http://creativecommons.org/licenses/by/2.0), which permits unrestricted use, distribution, and reproduction in any medium, provided the original work is properly cited.

\begin{abstract}
Background: Alternative splicing and polyadenylation are important mechanisms for creating the proteomic diversity necessary for the nervous system to fulfill its specialized functions. The contribution of alternative splicing to proteomic diversity in the nervous system has been well documented, whereas the role of alternative polyadenylation in this process is less well understood. Since the CstF-64 polyadenylation protein is known to be an important regulator of tissue-specific polyadenylation, we examined its expression in brain and other organs.

Results: We discovered several closely related splice variants of CstF-64 - collectively called $\beta C$ stF-64 - that could potentially contribute to proteomic diversity in the nervous system. The $\beta$ CstF-64 splice variants are found predominantly in the brains of several vertebrate species including mice and humans. The major $\beta$ CstF- 64 variant mRNA is generated by inclusion of two alternate exons (that we call exons 8.1 and 8.2) found between exons 8 and 9 of the CstF-64 gene, and contains an additional 147 nucleotides, encoding 49 additional amino acids. Some variants of $\beta C$ stF-64 contain only the first alternate exon (exon 8.I) while other variants contain both alternate exons (8.I and 8.2). In mice, the predominant form of $\beta$ CstF- 64 also contains a deletion of 78 nucleotides from exon 9 , although that variant is not seen in any other species examined, including rats. Immunoblot and 2D-PAGE analyses of mouse nuclear extracts indicate that a protein corresponding to $\beta$ CstF-64 is expressed in brain at approximately equal levels to CstF-64. Since $\beta C$ stF-64 splice variant family members were found in the brains of all vertebrate species examined (including turtles and fish), this suggests that $\beta C$ stF- 64 has an evolutionarily conserved function in these animals. $\beta$ CstF- 64 was present in both pre- and post-natal mice and in different regions of the nervous system, suggesting an important role for $\beta$ CstF-64 in neural gene expression throughout development. Finally, experiments in representative cell lines suggest that $\beta C$ stF-64 is expressed in neurons but not glia.
\end{abstract}

Conclusion: This is the first report of a family of splice variants encoding a key polyadenylation protein that is expressed in a nervous system-specific manner. We propose that $\beta$ CstF-64 contributes to proteomic diversity by regulating alternative polyadenylation of neural mRNAs. 


\section{Background}

Alternative mRNA processing is the mechanism by which multiple forms of mRNAs are generated from a common pre-mRNA via differential ligation of exons (alternative splicing, [1]), or differential 3' end site choice (alternative polyadenylation, [2]). Alternative mRNA processing contributes to proteomic diversity by generating protein isoforms that have different biochemical and structural properties $[3,4]$. Both of these processes are regulated in a tissue-specific manner, with the highest incidence occurring in the nervous system [5-7]. Not surprisingly, the processes of splicing and polyadenylation are coupled, leading to a high degree of interaction between the two processing machineries $[8,9]$. However, there have been only a few reports of alternative processing of mRNAs encoding critical proteins of the polyadenylation machinery: alternative splicing of poly(A) polymerase (PAP, [10]) and alternative splicing and polyadenylation of the 77 $\mathrm{kDa}$ subunit of the cleavage stimulation factor (CstF-77) in Drosophila [11] and mammals [12] have been reported. Alternatively spliced forms of these and other polyadenylation proteins could contribute to expansion of the proteome by promoting tissue-specific polyadenylation.

Like splicing, polyadenylation is an essential step in gene expression. The process of 3 ' end formation promotes transcription termination, mRNA export from the nucleus to the cytosol, mRNA stability, and translation initiation $[13,14]$. In addition to having a constitutive role in gene expression, polyadenylation also contributes to the generation of mRNA isoforms in a tissue-specific manner, particularly in the nervous system. For example, the mRNAs encoding $\beta$-adducin [15], huntingtin [16], amyloid precursor protein [17], ferritin heavy chain $[18,19]$, glutamate transporter EAAT2 [20], voltage-gated potassium channel [21], 68-kDa neurofilament [22], and many other proteins [23-28] have alternative polyadenylation signals that lead to production of nervous system-specific transcripts. Nervous system-specific coupling of alternative splicing and polyadenylation of mRNAs encoding calcitonin/calcitonin gene-related peptide [29], neural cell adhesion molecule [30], and vesl-1/homer1 [31] proteins lead to generation of tissue-specific protein isoforms from a common pre-mRNA. It has been speculated that use of alternative polyadenylation signals in these mRNAs may be due to expression of nervous system-specific polyadenylation factors [32], although none have been reported. CstF-64 is a key subunit of the polyadenylation complex that is known to function in regulation of polyadenylation [33,34]. Important domains of CstF-64 include an RNA-binding domain [35-38], CstF-77 interaction domain [39], proline/glycine-rich domain [36], MEARA repeat domain [40], and a conserved C-terminal domain [41]. Furthermore, a paralogous form of CstF-64, $\tau$ CstF-
64, was previously discovered in mouse male germ cells [42] and was found to be essential for normal spermatogenesis [43].

In this paper, we report the discovery of a family of alternatively spliced forms of the CstF-64 mRNA that are expressed in the nervous system of all vertebrate animals we have examined. These splice variants, which we collectively call $\beta$ CstF- 64 , are due to inclusion of one or two alternate exons between constitutive exons 8 and 9 . In mice, these alternate exons join to an alternative 3' splice site within exon 9. Mice express another minor splice variant, $\alpha$ CstF- 64 , which is formed by joining of exon 8 to the alternative 3 ' splice site in exon 9. However, the $\alpha$ CstF64 splice variant was not observed in any other species including rats or humans. All the $\beta \mathrm{CstF}-64$ splice variants are in-frame with the CstF- 64 coding region and encode up to 49 additional amino acids in the proline/glycinerich domain. The $\beta$ CstF- 64 splice variants are expressed in brains of many vertebrate species, including human and turtle, and the genome of simple vertebrates such as pufferfish and zebrafish contain homologous sequences. This leads us to hypothesize that the $\beta \mathrm{CstF}-64$ variants have an important evolutionarily conserved role in brain function. Other experiments suggest that $\beta \mathrm{CstF}-64$ is expressed predominantly in neurons, suggesting that it plays a role in expression of alternatively polyadenylated mRNAs important for neuronal function, thus contributing to proteomic diversity in the nervous system.

\section{Results \\ Alternatively spliced CstF-64 mRNAs are present in mouse brain}

Previous results from our laboratory indicated that CstF64 mRNA could be subject to alternative splicing (B. Dass and A. M. Wallace, unpublished). In order to investigate this possibility, we conducted an RT-PCR survey of CstF64 mRNA in mouse tissues using primers that together span the entire CstF-64 coding region (Figure 1). RNA was isolated from various mouse tissues as indicated and subjected to RT-PCR using primer pairs A, B, C and D that span exons $1-5,5-8,7-11$ and 9-14 respectively (Figure 1A). RT-PCR with primer pairs A, B, and D yielded a single PCR product in all tissues that corresponded to the reported form of CstF-64 (Figure $1 \mathrm{~B}$, panels a, b, d, lanes 1-8). This suggests little or no alternative splicing between exons 1-8 and 9-14. However, RT-PCR using primer pair $C$ (which spans exons $7-11$ ) resulted in multiple PCR products (Figure $1 \mathrm{~B}$, panel $\mathrm{c}$ ). Every mouse tissue examined showed the presence of the reported form of CstF-64 (595 bp) and a shorter splice variant that we named $\alpha$ CstF-64 (panel c, lanes 1-8). Cloning and sequencing of the $\alpha \mathrm{CstF}-64$ PCR product indicated that the shorter splice variant of CstF- 64 was generated by joining of exon 8 to an alternative 3 ' splice site in exon 9 


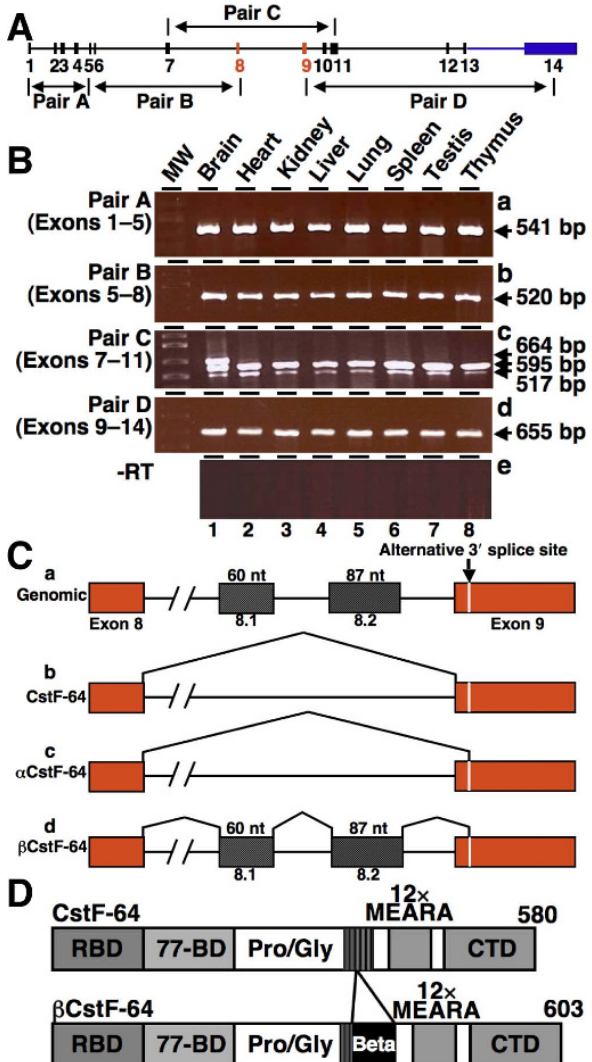

Figure I

An alternatively spliced mRNA of CstF-64 is present in mouse brain. A) Gene structure of CstF-64 and location of primer pairs. Boxes indicate exons; black line indicates introns; exons 8 and 9 are in red while all the other exons are black; the 3' UTR is blue. B) RT-PCR analysis of alternatively spliced mRNAs of CstF-64. RNA from indicated mouse tissues was subjected to RT-PCR using primer pairs $A, B, C$, and $\mathrm{D}$ (panels $\mathbf{a}, \mathbf{b}, \mathbf{c}$, and $\mathbf{d}$ respectively). Sizes of amplified products are indicated at the right. Panel e (-RT) denotes RT-PCR using primer pair $C$ with no reverse transcriptase added. C) Splicing patterns in CstF-64 mRNA. a) CstF-64 genomic structure showing exon 8,9 and the intervening intron. Boxes indicate exons and horizontal lines indicate introns; vertical lines indicate splicing patterns. The thin white line in exon 9 denotes the alternative 3' splice site. b) Splicing pattern of the regular form of CstF-64 mRNA. c) Splicing pattern of the shorter CstF-64 mRNA ( $\alpha$ CstF-64). d) Splicing pattern of $\beta$ CstF-64 mRNA. The sizes of the $\beta$ CstF-64-specific exons (8.I and 8.2) are indicated. D) Predicted domain structures of mouse CstF-64 and $\beta$ CstF-64. Shown are the RNA-binding domains (RBD, dark gray), the region of interaction with CstF-77 (77-BD, light gray), the proline/glycine domain (Pro/Gly, white box), MEARA repeat domain (I2× MEARA, dark gray) and the conserved C-terminal domain (CTD, light gray). The region within the Pro/Gly domain that contains a deletion of 26 amino acids is indicated by vertical lines, and the 49 amino acid $\beta$ CstF-64-specific domain is indicated by the black box. resulting in an mRNA molecule shorter by 78 nucleotides (Figure 1C). We reported this alternatively spliced form to GenBank as $\alpha$ CstF-64, variant 4 (GenBank: EU616681). The $\alpha$ CstF-64 splice variant is in frame with exon 9, and should result in a protein isoform that is shorter by 26 amino acids (not tested, but see Figure 2C). Since the $\alpha \mathrm{CstF}-64$ splice variant was not expressed in any other species we examined (see below), we chose not to characterize it further.

Surprisingly, using primer pair C, we found that brain had a larger PCR product ( $664 \mathrm{bp}$ ) that was absent from all the other tissues (Figure 1B, panel c, compare lane 1 to lane $2-8$ ). Cloning and sequencing of the larger PCR product revealed that it contained an additional 147 nucleotides between exon 8 and the $\alpha \mathrm{CstF}-64$ splice site within exon 9 (see Additional File 1 for nucleotide sequence). We named the alternate exons 8.1 and 8.2, and the longer brain-expressed variant CstF-64, $\beta$ CstF-64. The above form of mouse $\beta$ CstF- 64 containing exons $8.1,8.2$ and use of alternative 3 ' splice site was given the GenBank nomenclature $\beta$ CstF-64, variant 1 (GenBank: EU616682.1). We compared the sequence of the $\beta \mathrm{CstF}-64$ variant region to the mouse genome and determined that $\beta \mathrm{CstF}-64$ was generated by splicing of two alternate exons ( 8.1 and 8.2, Figure 1C) from the intronic region between exons 8 and 9 and selection of an alternative 3' splice site in exon 9. Exons 8.1 and 8.2 contained 60 and 87 nucleotides respectively. The alternative splicing patterns suggests that $\beta \mathrm{CstF}-64$ remains in-frame with the CstF-64 coding region, and that it encodes an expressed protein isoform of CstF-64. The predicted domain structure of the protein isoform encoded by $\beta$ CstF- 64 mRNA is illustrated in Figure 1D; this predicted protein isoform would lack 26 amino acids in the proline/glycine-rich domain of CstF64 encoded by exon 9 and contain an additional 49 amino acids encoded by exons 8.1 and 8.2 (Figure 1D). BLAST comparison of the brain-expressed $\beta$ CstF- 64 domain to all sequences in GenBank revealed no other described proteins with similar features (not shown). Thus, these data suggest that mouse $\beta$ CstF- 64 mRNA encodes a protein in brain that is 23 amino acids longer than CstF-64, containing a unique 49 amino acid brainspecific domain.

\section{The $\beta$ CstF-64 protein isoform is expressed in mouse brain} In order to investigate whether the $\beta \mathrm{CstF}-64$ protein was expressed in mouse brain, antibodies were raised against 16 amino acids of the unique 49 amino acid domain of the protein (Materials and Methods). The specificity of the anti- $\beta$ CstF-64 antibody was tested using recombinant $\beta$ CstF-64 and CstF-64 proteins expressed in HeLa cells (Figure 2A). Plasmids encoding epitope-tagged (3XFLAG)- $\beta$ CstF-64 and CstF- 64 proteins were transfected into HeLa cells as described in "Materials and Methods". 


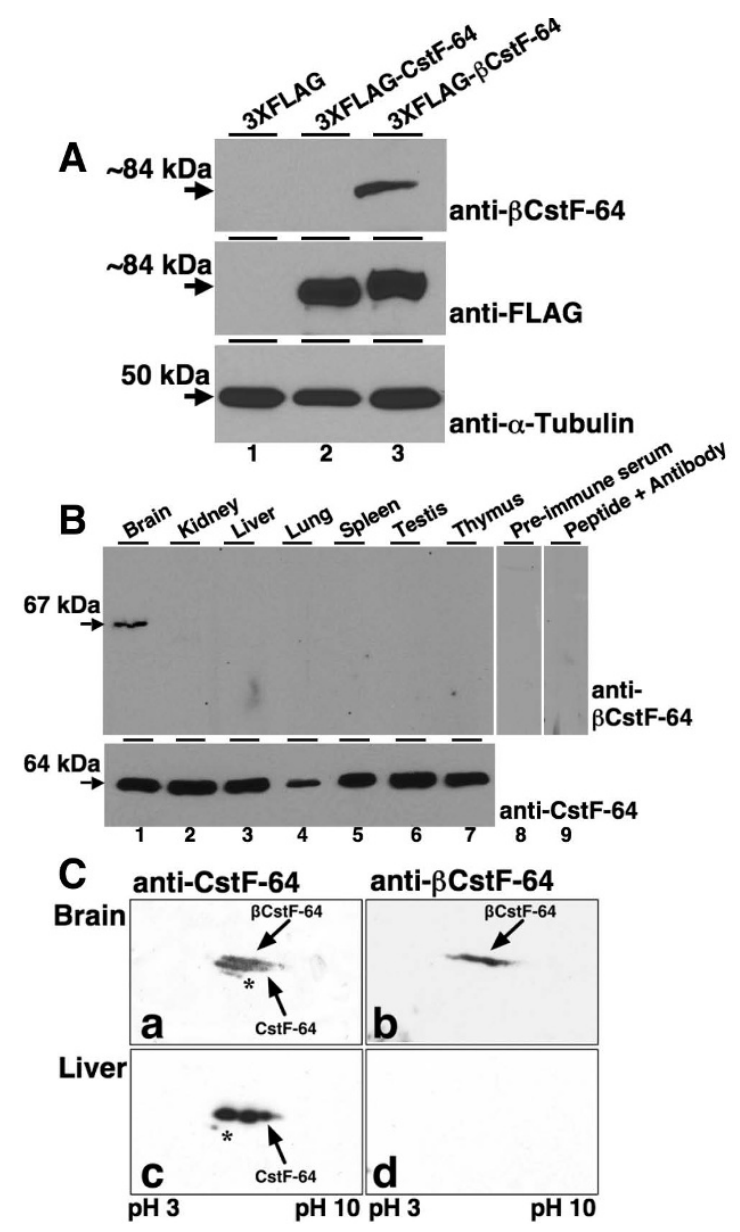

Figure 2

The $\beta$ CstF-64 protein is expressed predominantly in mouse brain. A) Whole cell extracts from HeLa cells transfected with $800 \mathrm{ng}$ of either 3XFLAG (lane I), 3XFLAGCstF-64 (lane 2) or 3XFLAG- $\beta$ CstF-64 (lane 3) were subjected to immunoblot analysis using anti- $\beta$ CstF-64 (upper panel), anti-FLAG (middle panel), or anti- $\alpha$-Tubulin (lower panel) antibodies. The apparent molecular weights of 3XFLAG-CstF-64, 3XFLAG- $\beta$ CstF- 64 , and tubulin are indicated at left. B) Protein immunoblot detection of $\beta$ CstF-64 in various mouse tissues. Nuclear extracts from indicated mouse tissues were subjected to immunoblot analysis using pre-immune serum (lane 8 ), peptide-blocked anti- $\beta$ CstF-64 antibody (lane 9), anti- $\beta$ CstF-64 antibody (upper panel, lanes I-7), or anti-CstF-64 antibody (lower panel, lanes I-7). The apparent molecular weights of CstF-64 and $\beta$ CstF-64 are indicated at left. C) 2D-PAGE analysis of mouse brain and liver nuclear extracts. Nuclear extracts were resolved in the first dimension by isoelectric focusing on a $\mathrm{pH} 3-10$ immobiline strip as indicated. The proteins were then resolved in the second dimension by denaturing PAGE, transferred to nitrocellulose membrane and probed with the anti-CstF-64 antibody (panels a and c) or anti- $\beta$ CstF-64 antibody (panels b and d). The arrows denote CstF-64 and $\beta$ CstF-64. The asterisk in panels $\mathbf{a}$ and $\mathbf{c}$ indicates a pattern that may be $\alpha$ CstF-64.
Whole cell lysates were prepared and subjected to SDSPAGE and immunoblot analysis using anti- $\beta$ CstF-64 (Figure 2A, top panel), anti-FLAG (Figure 2A, middle panel), and anti-tubulin antibodies (Figure $2 \mathrm{~A}$, bottom panel). This experiment demonstrated that the $\beta \mathrm{CstF}-64$ epitope was not detected in HeLa cells (lane 1) or in HeLa cells transfected with FLAG-CstF-64 (lane 2) but was expressed at the predicted size in HeLa cells transfected with FLAG$\beta C$ stF-64 (lane 3 ) suggesting that the anti- $\beta$ CstF-64 antibody could uniquely distinguish CstF-64 and $\beta$ CstF- 64 in mammalian cells.

Next, expression of $\beta$ CstF- 64 protein in various mouse tissues was investigated using the anti- $\beta \mathrm{CstF}-64$ antibody. Nuclear extracts from the indicated mouse tissues were subjected to immunoblot analysis using the anti- $\beta$ CstF- 64 and anti-CstF-64 antibodies (Figure 2B). Using the antiCstF-64 antibody, CstF-64 was detected in all tissues (Figure $2 \mathrm{~B}$, lower panel). In contrast, using the anti- $\beta$ CstF- 64 antibody, a protein of approximately $68 \mathrm{kDa}$ was detected in brain but not in other tissues examined (Figure 2B, upper panel, compare lanes 1 and 2-7). Detection of this protein was blocked by preincubation of the antibody with the 16 amino acid peptide that was used as an immunogen (lane 9). These results suggest that while the CstF64 protein is expressed in most or all tissues [44], the $\beta$ CstF-64 isoform is restricted to brain and probably other neural tissues.

\section{$\beta C s t F-64$ and CstF-64 protein levels are similar in mouse brain}

We wanted to investigate the relative levels of the CstF-64 and $\beta$ CstF- 64 proteins in mouse brain. Since we could not resolve $\beta$ CstF- 64 from CstF-64 proteins in a single dimension (Figure 2B and data not shown), 2D-PAGE analysis was employed to investigate this. Nuclear extracts from mouse brain and liver were resolved in the first dimension by isoelectric focusing and in the second dimension by SDS-PAGE [45]. Using the anti-CstF-64 antibody, at least two closely opposed patterns of spots were detected in brain (Figure 2C, panel a) while a single pattern of spots was detected in liver (Figure 2C, panel c). The basic to acidic range of spots most likely reflects differential phospohorylation of CstF-64 [36,46]. Identical blots were probed with the anti- $\beta$ CstF- 64 antibody, and only a single pattern of spots was detected in brain (Figure 2C, panel b), while no signal was detected in liver (panel d). Alignment of the anti-CstF-64 blot (panel a) with the anti$\beta$ CstF- 64 blot (panel b) confirmed that the anti- $\beta$ CstF- 64 pattern overlapped with the upper pattern in the antiCstF-64 blot. This suggested that only the upper pattern detected in brain by the anti-CstF- 64 antibody contained the $\beta \mathrm{CstF}-64$ epitope. Judging by the relative intensities of the two patterns of spots in brain (panel a), we estimate that the protein levels of CstF- 64 and $\beta$ CstF- 64 are approx- 
imately the same in mouse brain. Interestingly, a faint pattern of spots is seen in both brain and liver using the antiCstF-64 antibody (panels a and c, asterisks). We speculate that this might represent the mouse-specific $\alpha \mathrm{CstF}-64$ protein isoform.

\section{The $\beta$ CstF-64 family of splice variants is evolutionarily conserved}

In order to determine whether $\beta$ CstF-64 was also expressed in brains of other mammalian species, RT-PCR analysis was conducted using human RNA samples (Figure 3A). RT-PCR analysis using human-specific primers flanking exons 8 and 9 (Table 1) indicated that CstF-64 mRNA was present in brain, liver and testis (Figure 3A, lanes 1-3). A larger PCR product was detected in human brain RNA (Figure 3A, lane 1). Smaller amounts of this product were also detected in human testis (lane 3 ). Cloning and sequencing of the larger PCR product from human brain revealed a product that contained only exon 8.1 and lacked exon 8.2 and use of the alternative 3 ' splice site. This demonstrated that the alternatively spliced $\beta C$ StF-64 isoform in human brain differed from that in mice. We named this isoform, $\beta$ CstF-64 variant 2 (GenBank: EU616679.1). However, EST database searches (NCBI) showed that mRNAs containing exon 8.1 joined to exon 8.2 were found in humans (Accession numbers:

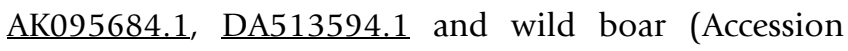
number: AJ959057.1). Therefore, using primers specific to the predicted exon 8.2, we detected mRNAs containing this exon in humans (data not shown). We have never observed a $\beta$ CstF-64 variant mRNA (either by database searches or by cloning) containing exon 8 joined to exon 8.2 in either humans, mice or any other mammals. The $\beta C$ stF-64 mRNA that contained both exons 8.1 and 8.2 but lacked use of the alternative 3' splice site in exon 9 was given the GenBank nomenclature, $\beta \mathrm{CstF}-64$ variant 3 (GenBank: EU616680.1). These data suggested that both $\beta$ CstF-64 variant 2 (exon 8.1 only) and $\beta$ CstF- 64 variant 3 (exon 8.1 and 8.2 ) are expressed in human brain. The $\beta C$ stF-64 variant 3 mRNA was also detected in the brains of the mouse species Mus spretus and in rats (data not shown). No product corresponding to $\alpha \mathrm{CstF}-64$ was seen in any of the human samples (lanes 1-3).

We wanted to determine whether the $\beta$ CstF-64 variant family was restricted to mammals, or could be detected in other vertebrate species. Therefore, RT-PCR analysis was conducted using RNA from brain and liver of turtle (Trachemys scripta elegans). RT-PCR products corresponding to $\beta C s t F-64$ mRNA were detected in turtle brain (Figure 3, lane 7) but not liver (Figure 3, lane 8). Cloning and sequencing of the band revealed that its splicing pattern

Table I: Primers used in detection and analysis of CstF-64 splice variants

\begin{tabular}{|c|c|c|}
\hline Species & Primer Name & Primer Sequence \\
\hline Mouse & Pair A & $\begin{array}{l}\text { 5' GGGTGAGCCATGGCGGGTT 3' } \\
\text { 5' CTGTTCTGGTGGAAGACTGGCAA 3' }\end{array}$ \\
\hline Mouse & Pair B & $\begin{array}{l}\text { 5' CCCCAGGAAGCACGAAACA 3' } \\
\text { 5' CCTCGTTCCATGGGCACTG 3' }\end{array}$ \\
\hline Mouse, Rat & Pair C & $\begin{array}{l}\text { 5' CAATGGCGCACCTCCTATGATG 3' } \\
\text { 5' GGCACGGGCTTCCAGTCCT 3' }\end{array}$ \\
\hline Mouse & Pair D & $\begin{array}{l}\text { 5' GATTAGATGCACGGGGGATGGA 3' } \\
\text { 5' TGGAGCAATGGCGATGTAAGACC 3' }\end{array}$ \\
\hline Human & Pair C & $\begin{array}{l}\text { 5' CCCCTCAGGCCCAGTCTTTG 3' } \\
\text { 5' TGGCCCTCCCCTCAGTTCAT 3' }\end{array}$ \\
\hline Turtle & Pair C & $\begin{array}{l}\text { 5' GACAGATGCCAGCCTCCGTAGC 3' } \\
\text { 5' CCATTGGTCCTCCCCTCATTTCAT 3' }\end{array}$ \\
\hline Mouse & $x$ & 5' CAATGGCGCACCTCCTATGATG 3' \\
\hline Mouse & Y & 5' TTCCACCTTGCATGCTTGCTC 3 \\
\hline Mouse & $\mathrm{E}$ & 5 ' GATCTATGGCGGGTTTGCCAGTGAG 3' \\
\hline Mouse & $\mathrm{F}$ & 5 ' TCTAGATCAAGGTGCCCCAGTGGATTTC 3 \\
\hline
\end{tabular}

Nucleotide sequences of the various primer pairs used in this study are indicated at right. The names of primer pairs along with their corresponding species are indicated at left in the table. 


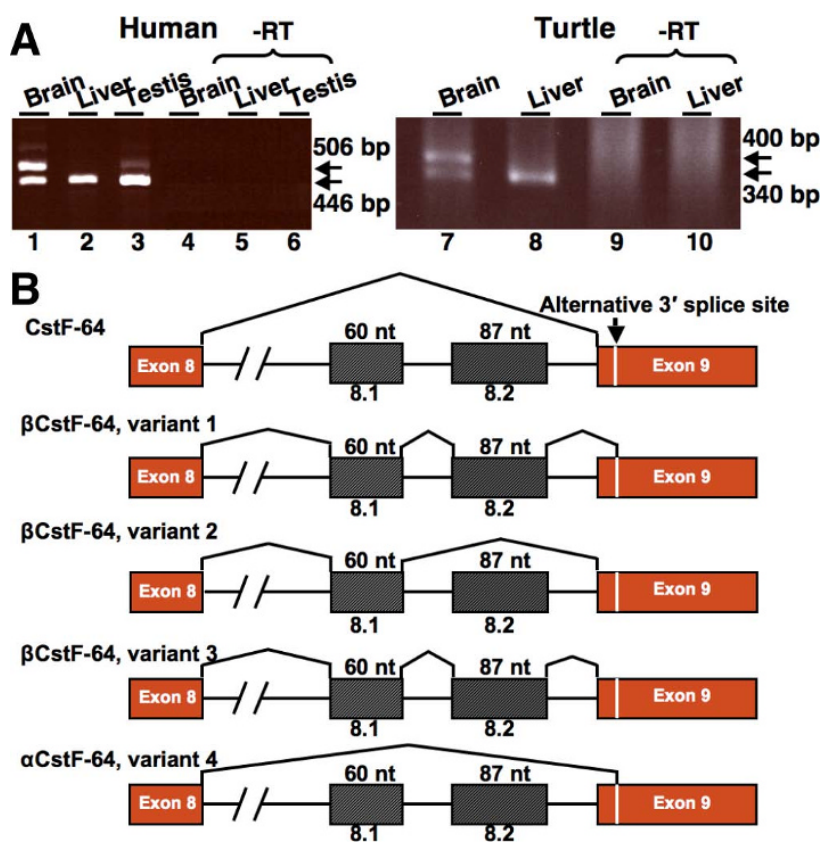

Figure 3

The $\beta$ CstF-64 family of splice variants is evolutionarily conserved in vertebrates. A) RT-PCR analysis of human and turtle CstF-64 mRNAs. Human brain, liver, and testis RNAs were subjected to RT-PCR using primers flanking exons 8 and 9 of the human CstF-64 gene (lanes I-6). RNA isolated from brain and liver of adult turtle was subjected to RT-PCR analysis using primers flanking exons 8 and 9 of the turtle CstF-64 gene (lanes 7-10). RT-PCR with no reverse transcriptase (-RT) is shown in lanes 4-6 (left panel) and lanes 9, 10 (right panel). B) Alternative splicing patterns of CstF-64 in various animal species. The splicing pattern of CstF-64 is shown on top. The distributions of the $\beta$ CstF-64 variant mRNAs in different animal species is as follows: $\beta$ CstF-64, variant I: Mus musculus; $\beta$ CstF-64, variant 2 : chimpanzee (Pan troglodytes), bovine (Bos taurus), dog (Canis familiaris), thirteen-lined ground squirrel (Spermophilus tridecemlineatus), gray short-tailed opossum (Monodelphis domestica), African clawed toad (Xenopus laevis), duck-billed platypus (Ornithorhynchus anatinus), zebrafish (Danio rerio), and pufferfish (Tetraodon nigroviridis); $\beta$ CstF-64, variant 3: Algerian mouse (Mus spretus), rat (Rattus norvegicus), human (Homo sapiens), pig (Sus scrofa domestica); $\beta$ CstF-64, variant 4: mouse (Mus musculus). The splicing patterns of $\beta$ CstF-64 mRNA in mice (Mus musculus and Mus spretus), rat, human, ground squirrel, short-tailed opossum, alligator, and turtle were determined from cloning and sequencing of RT-PCR products. The splicing patterns for chimpanzee, bovine, dog, toad, platypus, zebrafish, and pufferfish were obtained by searches of EST and protein databases utilizing BLASTX and BLASTP programs (not shown).

was similar to $\beta$ CstF-64 variant 2 mRNA observed in human (Figure 3B). RT-PCR analysis suggested that $\beta C$ stF64 mRNA with a splicing pattern similar to that observed in turtle was also present in ground squirrel, alligator, and Monodelphis (not shown, summarized in Figure 3B).
Database searches using exons 8.1 and 8.2 as query were conducted to determine the distribution of $\beta \mathrm{CstF}-64$ in various animal species (Figure $3 \mathrm{~B}$ and Additional File 2). Comparison of mouse $\beta \mathrm{CstF}-64$ nucleotide sequence to the human genome showed that exon 8.1 was $98 \%$ identical whereas exon 8.2 was $96 \%$ identical. The nucleotide sequence of exons 8.1 and 8.2 were $96 \%$ identical when compared to that of rhesus monkey (Macaca mulatta). Additionally, survey of the whole-genome-shotgun sequence database at NCBI showed a high degree of conservation of exons 8.1 and 8.2 in the genomes of other mammalian species such as guinea pig, horse, cat, and others. Finally, using BLASTX and BLASTP searches at the NCBI database, amino acids encoded by exon 8.1 were predicted to be present in proteins from chimpanzee, cow, dog (eutherian mammals), platypus (monotreme), Xenopus (amphibian), pufferfish, and zebrafish (fish). We note that exon 8.2 was not explicitly observed in these species, but the incomplete nature of some of these genome data sets does not allow us to rule out its presence. The high degree of conservation in vertebrate animal species suggests an essential function for the $\beta \mathrm{CstF}-64$ family of splice variants in neural mRNA polyadenylation.

\section{$\beta$ CstF-64 mRNA is present throughout the mouse nervous system}

An RT-PCR approach was used to investigate the expression of $\beta$ CstF- 64 mRNA in mouse nervous system. RNA was isolated from mouse spinal cord and five different regions of the brain (cerebrum, cerebellum, brain stem, diencephalon and olfactory bulb). $\beta$ CstF-64 mRNA was detected in all five brain regions (Figure 4, lanes 3-7) and in spinal cord, but not in adrenal gland (Figure 4, lane 8), consistent with nervous system-predominant expression of $\beta$ CstF-64. RT-PCR of ribosomal S16 mRNA was used to assess RNA loading (Figure 4, lower panel).

\section{BCstF-64 mRNA is not present in glial cell lines examined}

We wanted to determine in which neural cell types (neurons or glia) $\beta$ CstF- 64 was expressed. For this, RNAs from various rodent and human neuronal and glial cell lines were subjected to RT-PCR using species-specific primers flanking exons 8 and 9 of the CstF-64 gene (Figure 5). The rat and human thyroid carcinoma cell lines (CA77, Figure $5 \mathrm{~A}$, lane 5 and TT, Figure 5B, lane 2) used in this study have a neuronal phenotype and are neuroendocrine in nature $[47,48]$. The PC-12 cell line (Figure 5A, lane 6) derived from rat adrenal chromaffin cells, is neuroendocrine in nature, and, when treated with nerve growth factor (NGF, lane 7), differentiates into a cell that resembles the sympathetic neurons in the peripheral nervous system [49]. $\beta$ CstF-64 mRNA was detected in CA77 (Figure 5A, lane 5), undifferentiated and NGF-differentiated PC-12 cell lines (Figure 5, lanes 6 and 7), TT (Figure 5B, lane 2), and SK neuroblastoma cell lines (Figure 5B, lane 3 ). Interestingly, the expression of $\beta \mathrm{CstF}-64$ mRNA appeared to 


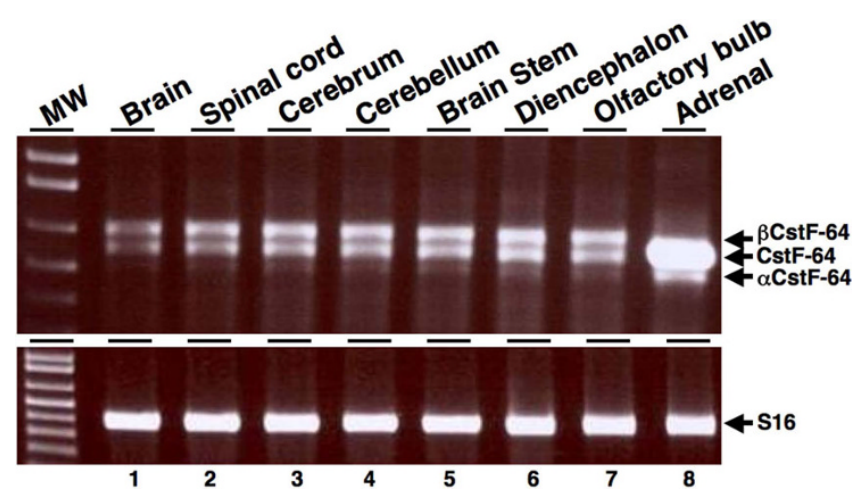

Figure 4

$\beta$ CstF-64 mRNA is present throughout the mouse nervous system. RT-PCR analysis was performed on RNA isolated from the spinal cord, adrenal gland and five regions of mouse brain using primer pair $C$ that flanks exons $7-1 \mathrm{I}$ of the CstF-64 gene. RT-PCR products were resolved by I\% agarose gel electrophoresis. RT-PCR of ribosomal SI6 mRNA (lower panel) was used a loading control.

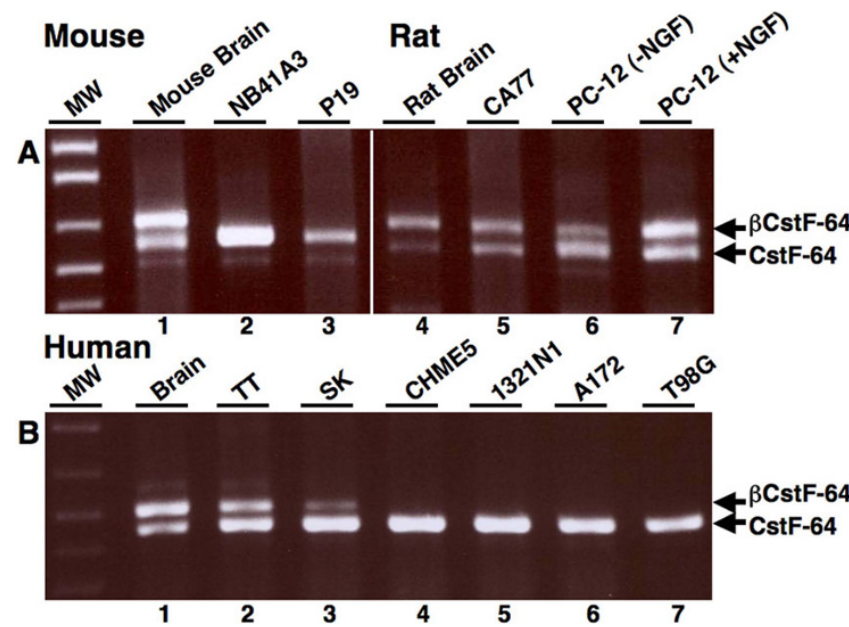

Figure 5

$\beta$ CstF-64 mRNA expression in neuronal and glial cell lines. RT-PCR analysis of $\beta$ CstF-64 using exon-specific primer pairs (Table $\mathrm{SI}$ ) from indicated rodent and human neuronal and glial cell lines. A) RT-PCR analysis of $\beta$ CstF-64 mRNA from mouse brain (lane I), NB4IA3 mouse neuroblastoma (lane 2), PI9 mouse embryonic carcinoma (lane 3), rat brain (lane 4), CA77 rat thyroid carcinoma (lane 5), PC12 rat pheochromocytoma (lane 6), or PC- 12 rat pheochromocytoma cells treated for 4 days with $50 \mathrm{ng} / \mathrm{mL}$ NGF (lane 7). B) RT-PCR analysis of $\beta$ CstF-64 mRNA from human brain (lane I), TT thyroid carcinoma (lane 2), SK neuroblastoma (lane 3), CHME5 microglia (lane 4), I32INI astrocytoma (lane 5), AI 72 astrocytoma (lane 6), or T98G glioblastoma cells (lane 7). increase in PC-12 cells treated with nerve growth factor (NGF, lane 7). $\beta$ CstF-64 mRNA was not detected in mouse P19 embryonic carcinoma cell line (Figure 5A, lane 3), and NB41A3 neuroblastoma cell line (Figure 5A, lane 2). In contrast, $\beta$ CstF- 64 was not detected in any of the human glial cell lines tested (Figure 5B, lanes 4-7), suggesting that $\beta$ CstF- 64 expression might be restricted to neurons in the nervous system.

\section{$\beta$ CstF-64 mRNA is present in mice of all ages}

We wanted to investigate the developmental expression of $\beta$ CstF-64 in mice (Figure 6). For this experiment, female mice mated with male mice were examined for the presence of copulatory plugs. The day copulatory plugs were found, embryos were designated as day 1 post-coitum (1 dpc). Newborn mice were designated as day 1 postpartum ( $1 \mathrm{dpp}$ ) and mice at $42 \mathrm{dpp}$ were designated as adult. RNA from the brains of embryos at 15, 19, $21 \mathrm{dpc}, 1 \mathrm{dpp}$, and adult male mice was subjected to RT-PCR analysis using primers flanking exons 8 and 9 of the CstF-64 gene. The mRNAs corresponding to $\alpha$ CstF-64, CstF- 64 and $\beta$ CstF-64 were detected at 15, 19, and $21 \mathrm{dpc}, 1 \mathrm{dpp}$, and adult mice (Figure 6, lane 1-5). RT-PCR of ribosomal S16 mRNA was used to assess RNA loading (Figure 6, lower panel). $\beta$ CstF64 mRNA was also detected in male and female mice of ages 1 dpp, 5 dpp, 7 dpp, 9 dpp, 13 dpp, 15 and 42 dpp at approximately equal levels as CstF-64 mRNA (data not shown).

\section{Discussion and conclusion}

While surveying the CstF-64 mRNA for evidence of alternative splicing, we made the discovery of a family of alternatively spliced forms, collectively called $\beta$ CstF- 64 , that

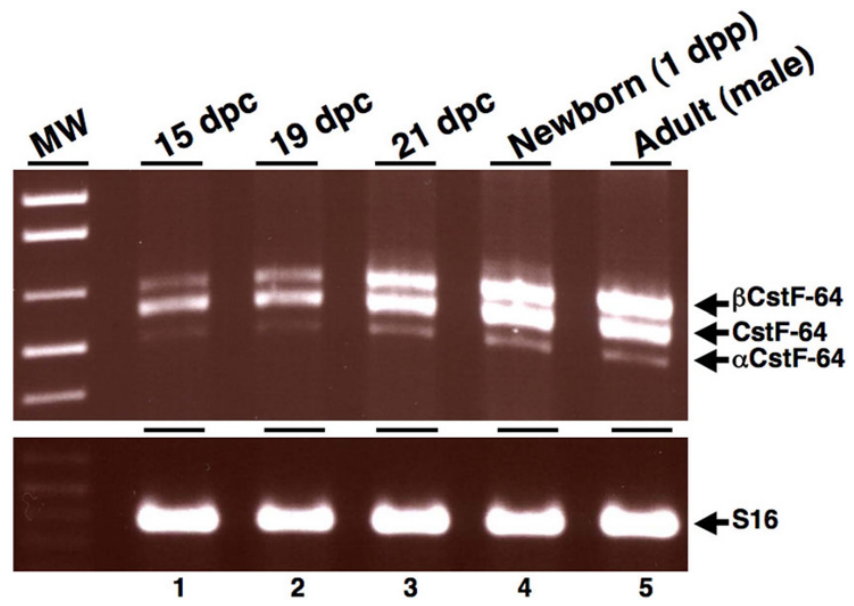

Figure 6

$\beta$ CstF-64 mRNA is present in mice of all ages. RT-PCR analysis using RNA isolated from brain of day 15 embryo (lane I), day 19 embryo (lane 2), day 21 embryo (lane 3 ), newborn mice (I dpp, lane 4), and adult male mice (lane 5). RT-PCR of ribosomal SI 6 mRNA (lower panel) was used a loading control. 
were found exclusively in the brain and spinal cord of mice and other vertebrate animal species (Figures 1, 3). In mice, the predominant form of these splice variants includes two alternate exons (8.1 and 8.2) joined to an alternative 3 ' splice site in exon 9, encoding an additional 49 amino acids while deleting 26 amino acids from exon 9. Interestingly, a smaller splice variant, $\alpha \mathrm{CstF}-64$, which was expressed widely in mice (Figure $1 \mathrm{~B}$ ), was not observed in non-rodent species, suggesting it arose independently of the $\beta$ CstF- 64 variants. We summarized the nomenclature of these isoforms in Figure 3B. Since our data demonstrate that the $\beta \mathrm{CstF}-64$ protein is expressed in mice (Figure 2), and that the $\beta$ CstF-64 mRNA family is present in all vertebrate species examined, we propose that the $\beta$ CstF- 64 protein variant has an ancient and essential role in neural function. We hypothesize that $\beta C$ CstF-64 regulates alternative polyadenylation in these tissues, leading to greater diversity of neural gene expression.

A survey of the EST and protein databases (BLASTX and BLASTP programs) indicated that exon 8.1 was predicted to be present in mammalian and non-mammalian genomes. In contrast, a similar analysis showed that exon 8.2 was predicted to be present only in mammalian genomes. This suggests the possibility that exon 8.2 appeared after the divergence of mammals and other vertebrates, and might serve to supplement the function of the more ancient exon 8.1. We also found that the intronic regions between exons 8.1, 8.2 and 9 were highly conserved between mice and humans (84\% identity). Since the intronic regions and exons 8.1 and 8.2 likely contain splicing regulatory sequences [50], this supports the notion that the region between exons 8 and 9 is a "hot spot" for alternative splicing during evolution. We failed to detect $\beta$ CstF-64 exonic sequences in invertebrates such as Drosophila [51] and the simple chordate Ciona intestinalis [52], leading us to hypothesize that $\beta$ CstF- 64 has a role in aspects of mRNA polyadenylation that are specific to higher vertebrates, perhaps being critical for important features of neural functions such as myelination [53].

In surveying rodent and human neuronal and glial cell lines (Figure 5), we obtained data suggesting that $\beta \mathrm{CstF}-$ 64 expression was restricted to neurons in the nervous system. Even though we did not detect $\beta$ CstF- 64 in any of the glial cell lines examined, we do not rule out the possibility that it might be expressed in normal glial cells. Detailed immunohistochemical analysis of brain slices will provide a better understanding of $\beta \mathrm{CstF}-64$ expression in neurons and glia.

We obtained data suggesting that $\beta$ CstF- 64 was expressed in cell lines that represent neural progenitor cells and are neuroendocrine in nature (Figure 5). For example, we found evidence for $\beta \mathrm{CstF}-64$ expression in neural-crest derived cell lines such as CA-77 and thyroid carcinoma cells. We also obtained data suggesting that while $\beta$ CstF64 was expressed in undifferentiated and NGF-differentiated PC-12 cell lines (Figure 5), it was not expressed in adrenal gland (Figure 4). PC-12 cells, unlike adrenal chromaffin cells, are thought to possess the pluripotency of primitive progenitor cells such as neural crest cells. These data support the hypothesis that $\beta \mathrm{CstF}-64$ is important for neuronal gene expression.

What might be the function of $\beta$ CstF- 64 in the nervous system? Our finding that $\beta$ CstF- 64 was expressed throughout the nervous system and at different stages of development suggests that it might be important for global neural gene expression and brain function. Several studies have indicated that many neural mRNAs have multiple polyadenylation sites in their 3' UTRs or use sites that differ from those in other tissues. Many of these mRNAs have regulatory elements surrounding the alternative polyadenylation sites. Differential use of polyadenylation sites within the 3' UTR has been shown to regulate translation [17], stability [54], and rate of transcription [54] of these neural mRNAs. It has been hypothesized that tissue-specific use of polyadenylation sites in these mRNAs might be due to the expression of nervous system-specific polyadenylation factors [15]. Our discovery of the nervous system-specific $\beta$ CstF-64 splice variant supports the above hypothesis, and leads us to propose that $\beta$ CstF- 64 might recognize alternative polyadenylation signals in these mRNAs contributing to their neural-specific expression. It is possible that $\beta$ CstF- 64 might also contribute to the generation of protein isoforms specific to the nervous system by use of internal poly(A) sites found in many neural mRNAs [29-31].

How might $\beta$ CstF-64 function in neural mRNA polyadenylation? In one model, $\beta$ CstF- 64 might recognize RNA sequence elements that are specific to nervous systemexpressed polyadenylation sites. A similar model has been proposed for the testis-expressed $\tau$ CstF- 64 variant $[35,55]$. A bioinformatic survey revealed that the downstream $\mathrm{U} /$ GU-rich sequences in the pre-mRNA are enriched in nervous system-specific transcripts, lending plausibility to this model [6]. How might $\beta$ CstF-64 recognize these variant RNA sequence elements if its RNA-binding domain is the same as CstF-64? The insertion in $\beta$ CstF-64 in the proline/ glycine-rich domain might alter the conformation of that domain which might in turn affect the conformation of the RNA-binding domain and alter the RNA binding specificities of $\beta$ CstF- 64 or otherwise affect the overall rate of polyadenylation [56]. In another model, the insertion of the $\beta$ CstF-64 domain might alter the overall structure of the CstF complex (for example, its dimerization, [57]), affecting its interaction with CPSF or other components of the polyadenylation machinery. 
For a final model, we note that the 49 amino acid $\beta$ CstF64 domain is not similar to any other known protein domains (data not shown). Based on this, we speculate that the $\beta$ CstF-64 domain might be a previously undescribed protein-protein interaction domain. It is possible that $\beta \mathrm{CstF}-64$ interacts with as-of-yet unknown nervous system-specific polyadenylation factors or brings about new interactions within the polyadenylation complex. It might also interact with the splicing machinery since splicing factors are known to modulate polyadenylation efficiency [58-60]. Any of these proposed interactions could help $\beta$ CstF- 64 in generating protein isoforms via recognition of internal intronic and exonic poly(A) sites thus increasing proteomic diversity in the nervous system.

\section{Methods}

\section{Animals and RNA Samples}

All animal studies were conducted in accordance with the National Institutes of Health guidelines and all protocols were approved by the TTUHSC Institutional Animal Care and Use Committee. Tissues from adult male and female CD-1 mice (Charles River Laboratories, Wilmington, DE) were dissected post-euthanasia and stored in RNAlater (Ambion, Austin, TX). Brains from mouse embryos at days 15, 19, and 21 days post-coitus (dpc) were dissected from the gravid females post-euthanasia. The first day on which a vaginal plug was observed in the female, after mating, was considered as $1 \mathrm{dpc}$. Human brain, liver and testis RNA samples purchased from Ambion (Austin, TX) were from single individuals. Slider turtle (Trachemys scripta elegans) brain and liver RNA and 13-lined ground squirrel (Spermophilus tridecemlineatus) brain RNA were obtained from B. Dass (TTUHSC, Lubbock, TX) and Alligator (Alligator mississippiensis) custom cDNA library was a gift from Phil Hartig (US-EPA). Monodelphis tissues were provided by Janice MacRossin and John L. VandeBerg (Southwest Foundation for Biomedical Research).

\section{RNA Analysis and RT-PCR}

Different regions of the brain and indicated mouse tissues were dissected, total RNA extracted by using TRIzol reagent (Invitrogen, Carlsbad, CA), and treated with TurboDNase (Ambion). Equal amounts of DNased RNA (4 $\mu \mathrm{g})$ from each tissue sample were used to synthesize cDNA using SuperScriptII Reverse Transcriptase and oligo-dT according to the manufacturer's protocol (Invitrogen). Polymerase chain reaction (PCR, 30 cycles) was conducted using the indicated primers (see Table 1) in an Air Thermocycler (Idaho Technologies, Salt Lake City, UT). Amplicons were resolved by electrophoresis on a 1\% agarose gel electrophoresis. Ethidium bromide stained RTPCR products were excised from the gel (Qiagen, Valencia, $\mathrm{CA}$ ), cloned using Topo II system (Invitrogen), and identified by DNA sequence analysis.

\section{Cloning and Plasmids}

The plasmid encoding full length $\beta \mathrm{CstF}-64$ fused to the FLAG epitope tag [61] (3XFLAG- $\beta$ CstF-64) was created as follows: mouse brain cDNA was subjected to RT-PCR using primers $\mathrm{X}$ and $\mathrm{Y}$ containing HinDIII, KpnI restriction sites (Table 1) and ligated into a 3X-HA vector encoding full length CstF-64 (kind gift of K. W. McMahon) digested by the same enzymes. Note that this vector also contained the MS2 RNA binding domain though that did not interfere with its expression or antigenicity. The 3XFLAG- $\beta$ CstF-64 plasmid was created by amplifying full length $\beta$ CstF-64 using primers $\mathrm{E}$ and $\mathrm{F}$ containing $\mathrm{XbaI}$, BglII sites (Table 1 ) using $3 \mathrm{X}-\mathrm{HA}-\beta \mathrm{CstF}-64$ as template. This fragment was digested with $\mathrm{Xba} \mathrm{I}$ and Bgl II, and ligated into a similarly digested 3XFLAG-CstF-64 vector. The cloning scheme of 3XFLAG-CstF-64 is described in [61].

\section{Cell Culture and Transfections}

HeLa cells were grown at $37^{\circ} \mathrm{C}$ in $5 \% \mathrm{CO}_{2}$ in Dulbecco's Minimal Eagle Media (CellGro, Manassas, VA) containing $10 \%$ cosmic calf serum and $1 \%$ penicillin/streptomycin (Gibco). HeLa cells were seeded in 6-well plates at a density of 300,000 cells/well and transfected the next day with $800 \mathrm{ng}$ of the indicated plasmids using Lipofectamine (Invitrogen). Cells were harvested 48 hours post-transfection by rinsing with ice-cold PBS, and lysed in SDS loading buffer [62].

\section{Antibodies}

The following antibodies were used in this study: anti- $\alpha$ tubulin and anti-FLAG antibodies (Sigma, St. Louis, MO); anti-CstF-64 antibody (3A7, [44]) and anti- $\beta$ CstF-64 custom antibody generated in rabbits by Genscript Corporation (Piscataway, NJ) by injection of the peptide GPAGPASIERVQGQRT representing part of the $\beta \mathrm{CstF}-64$ insert.

\section{Protein Analysis}

For protein analysis, nuclear extract was prepared from indicated mouse tissues [63], boiled and sonicated in SDS loading buffer [62]. Protein concentration was measured using the bicinchoninic acid (Pierce, Rockford, IL) assay. Equal amounts of protein from the indicated tissues (20 $\mu \mathrm{g})$ or HeLa cells were resolved by $10 \%$ SDS-PAGE, and transferred to nitrocellulose membranes for immunoblotting. For immunoblotting using anti-CstF-64 antibody, membranes were blocked with Tris-buffered saline containing $0.2 \%$ Tween-20 (TBST) with $2 \%$ nonfat dry milk (TBST) for 2 hours and treated with anti-CstF-64 antibody [44] at a dilution of 1:50. For immunoblotting using anti$\beta$ CstF-64 antibody, the membrane was first treated with $0.2 \mathrm{mM}$ glycine-HCl, pH 2.6 for 30 minutes at room temperature, and rinsed extensively with TBST. Pre-treatment with glycine at low $\mathrm{pH}$ was found to enhance the effectiveness of this antibody while decreasing non-specific back- 
ground. The membrane was then blocked with $2 \%$ nonfat dry milk in TBST for 2 hours. The anti- $\beta$ CstF- 64 antibody was used at a dilution of 1:5000 in 2\% nonfat dry milk in TBST, $0.05 \%$ Empigen (Calbiochem, La Jolla, CA). In the peptide blocking experiment, the anti- $\beta \mathrm{CstF}-64$ antibody $(1 \mu \mathrm{g} / \mu \mathrm{L})$ was incubated with 10 fold excess peptide (10 $\mu \mathrm{g}$ ) in $2 \%$ nonfat dry milk in TBST for 2 hours at room temperature before addition to the membrane. Membranes were subsequently treated with horseradish peroxidase-conjugated goat anti-mouse and anti-rabbit IgG (1:2500) and immunoreactive bands were visualized by chemiluminescence using the Pierce SuperSignal kit (Rockford, IL).

\section{Two dimensional-PAGE Analysis}

Nuclear extracts from mouse brain and liver were prepared as described above. Protein concentration was estimated by BCA assay. $392 \mu \mathrm{L}$ of rehydration buffer $(9 \mathrm{M}$ urea, 2\% Triton X-100), $8 \mu \mathrm{L}$ IPG buffer (pH 4-7) (Amersham Biosciences, Piscataway, NJ) and $0.5 \mu \mathrm{L} 1 \mathrm{M}$ DTT was added to $80 \mu \mathrm{g}$ of nuclear protein. The mix was layered over a $7 \mathrm{~cm} \mathrm{pH} \mathrm{3-10} \mathrm{immobiline} \mathrm{strip} \mathrm{(Amersham}$ Biosciences) and allowed to rehydrate for 16 hours. The strips were then subjected to isoelectric focusing at the following voltages: $200 \mathrm{~V}$ for 1 minute, $500 \mathrm{~V}$ for 20 minutes, $1000,1500,2000,2500 \mathrm{~V}$ for 20 minutes and $3500 \mathrm{~V}$ for 60 minutes. The strip was equilibrated in equilibration solution $(20 \%$ SDS, $50 \mathrm{mM}$ Tris, $\mathrm{pH} 8.8,6 \mathrm{M}$ urea, 30\% glycerol) containing $65 \mathrm{mM}$ DTT but no iodoacetamide for 15 minutes and then in equilibration solution containing $135 \mathrm{mM}$ iodoacetamide but no DTT for $15 \mathrm{~min}$ utes. The strip was then placed horizontally on a $10 \%$ SDS gel, subjected to denaturing PAGE and immunoblot analysis.

\section{Authors' contributions}

GSS conducted all experiments except the ones described below and wrote the manuscript. PWC conducted all the dissections described in the paper and helped analyze data. BD provided RNA from slider turtle and sequence information for CstF-64 from various animal species. CCM conceived the study, lent expert guidance, and provided critical comments for developing the manuscript. All authors read and approved the final manuscript.

\section{Additional material}

\section{Additional file 1}

Nucleotide and amino acid sequence of mouse $\beta C s t F-64$. The nucleotide sequence of exons 8.1 and 8.2 of mouse $\beta C$ CstF-64 (accession numbers provided) and their corresponding amino acid sequences are indicated. The nucleotide and amino acid sequences of the region deleted from exon 9 via use of the alternative 3' splice site are also shown. Click here for file

[http://www.biomedcentral.com/content/supplementary/14712199-10-22-S1.doc]

\section{Additional file 2}

Multiple sequence alignment of the 50 amino acid $\beta C$ st $F-64$ sequence from various animal species. The amino acid sequences of $\beta C s t F-64$ from mouse, rat, human, turtle, ground squirrel, alligator and monodelphis were predicted from cloning and in silico translation of RT-PCR products while the rest were determined by searching EST and protein databases at NCBI. Multiple sequence alignment was determined by ClustalW using sequences with the following accession numbers:

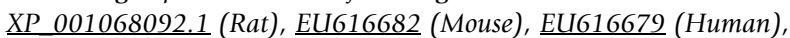
AJ959057.1 (Wild boar), XP 529072 (Chimpanzee), Ground Squirrel (B. Dass, unpublished), AAI1265544 (Cow), XP 549135 (Dog), Monodelphis (B. Dass, unpublished), XP 001513073 (Platypus), Alligator (B. Dass, unpublished), Turtle (G. Shankarling and B. Dass, unpublished), NP 001080179.1 (Xenopus), CAG09844.1 (Pufferfish), CU459168.8, CT027817.1 (Zebrafish). Boxed residues denote amino acids that differ from rodent. The various animal species included in this study are indicated on right.

Click here for file

[http://www.biomedcentral.com/content/supplementary/14712199-10-22-S2.pdf]

\section{Acknowledgements}

We thank Nathaly Cormier for help with 2D-PAGE analysis, Hua Lou, Peter Syapin, and Jose-Luis Redondo for providing RNA samples and cell lines, Wyatt McMahon and Andrew Hockert for providing plasmid constructs and Dr. Sandra Whelly for technical assistance and critical reading of manuscript. We also thank Phil Hartig (US-EPA) for providing the Alligator (Alligator mississippiensis) custom cDNA library and Janice MacRossin and John L. VandeBerg (Southwest Foundation for Biomedical Research) for providing Monodelphis tissue samples.

This work was supported by National Institutes of Health Grant 5 ROI HD37109-8, the South Plains Foundation, and the Texas Tech University Health Science Center SOM Seed Grant Program (to C.C.M).

\section{References}

I. Black DL: Mechanisms of alternative pre-messenger RNA splicing. Annu Rev Biochem 2003, 72:291-336.

2. Edwalds-Gilbert G, Veraldi KL, Milcarek C: Alternative poly(A) site selection in complex transcription units: means to an end? Nucleic Acids Res 1997, 25( 1 3):2547-256I.

3. Li Q, Lee JA, Black DL: Neuronal regulation of alternative premRNA splicing. Nat Rev Neurosci 2007, 8(I I):8|9-83I.

4. Stetefeld J, Ruegg MA: Structural and functional diversity generated by alternative mRNA splicing. Trends Biochem Sci 2005, 30(9):5I5-52I.

5. Yeo G, Holste D, Kreiman G, Burge CB: Variation in alternative splicing across human tissues. Genome Biol 2004, 5(I 0):R74.

6. Zhang $\mathrm{H}$, Lee JY, Tian B: Biased alternative polyadenylation in human tissues. Genome Biol 2005, 6( I 2):RI00.

7. Xu Q, Modrek B, Lee C: Genome-wide detection of tissue-specific alternative splicing in the human transcriptome. Nucleic Acids Res 2002, 30(I7):3754-3766.

8. Kyburz $A$, Friedlein A, Langen $H$, Keller $W$ : Direct interactions between subunits of CPSF and the U2 snRNP contribute to the coupling of pre-mRNA $3^{\prime}$ end processing and splicing. Mol Cell 2006, 23(2): 195-205.

9. Rigo F, Martinson HG: Functional coupling of last-intron splicing and 3 '-end processing to transcription in vitro: the poly(A) signal couples to splicing before committing to cleavage. Mol Cell Biol 2008, 28(2):849-862.

10. Zhao W, Manley JL: Complex alternative RNA processing generates an unexpected diversity of poly(A) polymerase isoforms. Mol Cell Biol 1996, 16(5):2378-2386. 
II. Mitchelson A, Simonelig M, Williams C, O'Hare K: Homology with Saccharomyces cerevisiae RNAI 4 suggests that phenotypic suppression in Drosophila melanogaster by suppressor of forked occurs at the level of RNA stability. Genes Dev 1993, 7(2):24l-249.

12. Pan Z, Zhang H, Hague LK, Lee JY, Lutz CS, Tian B: An intronic polyadenylation site in human and mouse CstF-77 genes suggests an evolutionarily conserved regulatory mechanism. Gene 2006, 366(2):325-334.

13. Edmonds M: A history of poly $\mathbf{A}$ sequences: from formation to factors to function. Prog Nucleic Acid Res Mol Biol 2002, 7 I:285-389.

14. Zhao J, Hyman L, Moore C: Formation of mRNA 3' ends in eukaryotes: mechanism, regulation, and interrelationships with other steps in mRNA synthesis. Microbiol Mol Biol Rev 1999, 63(2):405-445.

15. Costessi L, Devescovi G, Baralle FE, Muro AF: Brain-specific promoter and polyadenylation sites of the beta-adducin premRNA generate an unusually long 3'-UTR. Nucleic Acids Res 2006, 34(I):243-253.

16. Casanova E, Alonso-Llamazares A, Zamanillo D, Garate C, Calvo P, Chinchetru MA: Identification of a long huntingtin mRNA transcript in mouse brain. Brain Res 1996, 743(I-2):320-323.

17. de Sauvage F, Kruys V, Marinx O, Huez G, Octave JN: Alternative polyadenylation of the amyloid protein precursor mRNA regulates translation. Embo J 1992, I I (8):3099-3103.

18. Joshi JG, Fleming JT, Dhar M, Chauthaiwale V: A novel ferritin heavy chain messenger ribonucleic acid in the human brain. J Neurol Sci 1995, I 34(Suppl):52-56.

19. Percy ME, Wong S, Bauer S, Liaghati-Nasseri N, Perry MD, Chauthaiwale VM, Dhar M, Joshi JG: Iron metabolism and human ferritin heavy chain cDNA from adult brain with an elongated untranslated region: new findings and insights. Analyst 1998 , I 23(I):4I-50

20. Munch C, Schwalenstocker B, Hermann C, Cirovic S, Stamm S, Ludolph A, Meyer T: Differential RNA cleavage and polyadenylation of the glutamate transporter EAAT2 in the human brain. Brain Res Mol Brain Res 2000, 80(2):244-25I.

21. Jang GM, Tanaka BS, Gutman GA, Goldin AL, Semler BL: Alternative polyadenylation signals in the $3^{\prime}$ non-coding region of a voltage-gated potassium channel gene are major determinants of mRNA isoform expression. Gene 2008, 408(I2): $133-145$.

22. Nakahira K, Ikenaka K, Wada K, Tamura T, Furuichi T, Mikoshiba K: Structure of the $68-\mathrm{kDa}$ neurofilament gene and regulation of its expression. I Biol Chem 1990, 265(32): | 9786-1979|.

23. Ihara H, Tsutsuki H, Ida T, Kozaki S, Tsuyama S, Moss J: Alternative polyadenylation sites of human endothelial nitric oxide synthase mRNA. Biochem Biophys Res Commun 2007, 363(I): |46-I 52

24. Drews VL, Lieberman AP, Meisler MH: Multiple transcripts of sodium channel SCN8A $\left(\mathrm{Na}(\mathrm{V})\right.$ I.6) with alternative $5^{\prime}$ - and 3 -untranslated regions and initial characterization of the SCN8A promoter. Genomics 2005, 85(2):245-257.

25. Kobayashi K, Kurosawa Y, Fujita K, Nagatsu T: Human dopamine beta-hydroxylase gene: two mRNA types having different 3'terminal regions are produced through alternative polyadenylation. Nucleic Acids Res 1989, I7(3):1089-II02.

26. Pan MG, Rim C, Lu KP, Florio T, Stork PJ: Cloning and expression of two structurally distinct receptor-linked protein-tyrosine phosphatases generated by RNA processing from a single gene. J Biol Chem 1993, 268(26): 19284-19291.

27. Blasius R, Weber RG, Lichter P, Ogilvie A: A novel orphan G protein-coupled receptor primarily expressed in the brain is localized on human chromosomal band 2 q2 I. J Neurochem 1998, 70(4): 1357-1365.

28. Harakall SA, Brandenburg CA, Gilmartin GA, May V, Braas KM Induction of multiple pituitary adenylate cyclase activating polypeptide (PACAP) transcripts through alternative cleavage and polyadenylation of proPACAP precursor mRNA. Ann N Y Acad Sci 1998, 865:367-374.

29. Zhou HL, Baraniak AP, Lou H: Role for Fox-I/Fox-2 in mediating the neuronal pathway of calcitonin/calcitonin gene-related peptide alternative RNA processing. Mol Cell Biol 2007 27(3):830-84I.

30. Barbas JA, Chaix JC, Steinmetz M, Goridis C: Differential splicing and alternative polyadenylation generates distinct NCAM transcripts and proteins in the mouse Embo I 1988 7(3):625-632

31. Niibori Y, Hayashi F, Hirai K, Matsui M, Inokuchi K: Alternative poly(A) site-selection regulates the production of alternatively spliced vesl-I/homer I isoforms that encode postsynaptic scaffolding proteins. Neurosci Res 2007, 57(3):399-4l0.

32. Lou H, Gagel RF: Alternative RNA processing - its role in regulating expression of calcitonin/calcitonin gene-related peptide. J Endocrinol 1998, I 56(3):401-405

33. Shell SA, Hesse C, Morris SM Jr, Milcarek C: Elevated levels of the 64-kDa cleavage stimulatory factor (CstF-64) in lipopolysaccharide-stimulated macrophages influence gene expression and induce alternative poly(A) site selection. J Biol Chem 2005, 280(48):39950-3996।

34. Takagaki Y, Seipelt RL, Peterson ML, Manley JL: The polyadenylation factor CstF-64 regulates alternative processing of IgM heavy chain pre-mRNA during $B$ cell differentiation. Cell 1996, 87(5):94|-952.

35. Monarez RR, MacDonald CC, Dass B: Polyadenylation proteins CstF-64 and tauCstF-64 exhibit differential binding affinities for RNA polymers. Biochem J 2007, 40 I(3):65 I-658.

36. Takagaki Y, MacDonald CC, Shenk T, Manley JL: The human 64$\mathbf{k D a}$ polyadenylylation factor contains a ribonucleoproteintype RNA binding domain and unusual auxiliary motifs. Proc Natl Acad Sci USA 1992, 89(4): | 403-| 407.

37. Perez Canadillas JM, Varani G: Recognition of GU-rich polyadenylation regulatory elements by human CstF-64 protein. Embo J 2003, 22(I I ):282I-2830.

38. Salisbury J, Hutchison KW, Graber JH: A multispecies comparison of the metazoan 3'-processing downstream elements and the CstF-64 RNA recognition motif. BMC Genomics 2006, 7:55.

39. Takagaki Y, Manley JL: Complex protein interactions within the human polyadenylation machinery identify a novel component. Mol Cell Biol 2000, 20(5): I5 I5-I525.

40. Richardson JM, McMahon KW, MacDonald CC, Makhatadze GI: MEARA sequence repeat of human CstF-64 polyadenylation factor is helical in solution. A spectroscopic and calorimetric study. Biochemistry 1999, 38(39): | 2869-| 2875.

4I. Qu X, Perez-Canadillas JM, Agrawal S, De Baecke J, Cheng H, Varani $\mathrm{G}$, Moore C: The C-terminal domains of vertebrate CstF-64 and its yeast orthologue Rna 5 form a new structure critical for mRNA 3'-end processing. I Biol Chem 2007 282(3):2101-2II5

42. Dass B, McMahon KW, Jenkins NA, Gilbert DJ, Copeland NG, MacDonald CC: The gene for a variant form of the polyadenylation protein CstF-64 is on chromosome 19 and is expressed in pachytene spermatocytes in mice. I Biol Chem 200I, 276( I I ):8044-8050.

43. Dass B, Tardif S, Park JY, Tian B, Weitlauf HM, Hess RA, Carnes K, Griswold MD, Small CL, Macdonald CC: Loss of polyadenylation protein tauCstF-64 causes spermatogenic defects and male infertility. Proc Natl Acad Sci USA 2007, I 04(5 I):20374-20379.

44. Wallace AM, Dass B, Ravnik SE, Tonk V, Jenkins NA, Gilbert DJ, Copeland NG, MacDonald CC: Two distinct forms of the 64,000 Mr protein of the cleavage stimulation factor are expressed in mouse male germ cells. Proc Natl Acad Sci USA 1999 , 96( I 2):6763-6768.

45. O'Farrell PH: High resolution two-dimensional electrophoresis of proteins. J Biol Chem 1975, 250:4007-402I.

46. Edwalds-Gilbert G, Milcarek C: Regulation of poly(A) site use during mouse B-cell development involves a change in the binding of a general polyadenylation factor in a B-cell stagespecific manner. Mol Cell Biol I 995, I 5(I I):6420-6429.

47. Russo AF, Lanigan TM, Sullivan BE: Neuronal properties of a thyroid C-cell line: partial repression by dexamethasone and retinoic acid. Mol Endocrinol 1992, 6(2):207-2 18.

48. Berger CL, de Bustros A, Roos BA, Leong SS, Mendelsohn G, Gesell MS, Baylin SB: Human medullary thyroid carcinoma in culture provides a model relating growth dynamics, endocrine cell differentiation, and tumor progression. J Clin Endocrinol Metab 1984, 59(2):338-343.

49. Greene LA, Tischler AS: Establishment of a noradrenergic clonal line of rat adrenal pheochromocytoma cells which respond to nerve growth factor. Proc Natl Acad Sci USA 1976, 73(7):2424-2428. 
50. Cartegni L, Wang J, Zhu Z, Zhang MQ, Krainer AR: ESEfinder: A web resource to identify exonic splicing enhancers. Nucleic Acids Res 2003, 3 I (13):3568-357I.

5I. Adams MD, Celniker SE, Holt RA, Evans CA, Gocayne JD, Amanatides PG, Scherer SE, Li PW, Hoskins RA, Galle RF, et al: The genome sequence of Drosophila melanogaster. Science 2000 , 287(546I):2|85-2|95.

52. Dehal P, Satou Y, Campbell RK, Chapman J, Degnan B, De Tomaso A, Davidson B, Di Gregorio A, Gelpke M, Goodstein DM, et al:: The draft genome of Ciona intestinalis: insights into chordate and vertebrate origins. Science 2002, 298(560I):2157-2I67.

53. Northcutt RG: Understanding vertebrate brain evolution. Integrative and Comparative Biology 2002, 42(4):743-746.

54. $\mathrm{Hu} X, \mathrm{Bi}$ J, Loh HH, Wei LN: Regulation of mouse kappa opioid receptor gene expression by different 3'-untranslated regions and the effect of retinoic acid. Mol Pharmacol 2002, 62(4):88I-887.

55. MacDonald CC, Redondo JL: Reexamining the polyadenylation signal: were we wrong about AAUAAA? Mol Cell Endocrinol 2002, I 90(I-2): I-8.

56. Chao LC, Jamil A, Kim SJ, Huang L, Martinson HG: Assembly of the cleavage and polyadenylation apparatus requires about 10 seconds in vivo and is faster for strong than for weak poly $(A)$ sites. Mol Cell Biol 1999, I 9(8):5588-5600.

57. Bai Y, Auperin TC, Chou CY, Chang GG, Manley JL, Tong L: Crystal structure of murine CstF-77: dimeric association and implications for polyadenylation of mRNA precursors. Mol Cell 2007, 25(6):863-875.

58. Castelo-Branco P, Furger A, Wollerton M, Smith C, Moreira A Proudfoot N: Polypyrimidine tract binding protein modulates efficiency of polyadenylation. Mol Cell Biol 2004, 24(10):4174-4I83.

59. Lou H, Gagel RF, Berget SM: An intron enhancer recognized by splicing factors activates polyadenylation. Genes Dev 1996 I0(2):208-219.

60. Lou H, Neugebauer KM, Gagel RF, Berget SM: Regulation of alternative polyadenylation by UI snRNPs and SRp20. Mol Cell Biol 1998, I 8(9):4977-4985.

61. Maciolek NL, McNally MT: Characterization of Rous sarcoma virus polyadenylation site use in vitro. Virology 2008 374(2):468-476

62. Laemmli UK: Cleavage of structural proteins during the assembly of the head of bacteriophage T4. Nature 1970, 227(5259):680-685.

63. Gorski K, Carneiro M, Schibler U: Tissue-specific in vitro transcription from the mouse albumin promoter. Cell 1986, 47(5):767-776.

Publish with Bio Med Central and every scientist can read your work free of charge

"BioMed Central will be the most significant development for disseminating the results of biomedical research in our lifetime. "

Sir Paul Nurse, Cancer Research UK

Your research papers will be:

- available free of charge to the entire biomedical community

- peer reviewed and published immediately upon acceptance

- cited in PubMed and archived on PubMed Central

- yours - you keep the copyright
BioMedcentral 